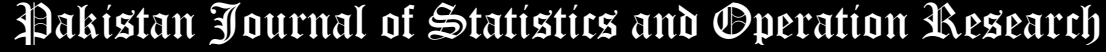

\section{Bivariate Transmuted Burr Distribution: Properties and Application}

\author{
Jumanah Ahmed Darwish ${ }^{1}$, Lutfiah Ismail Al turk ${ }^{2}$ and Muhammad Qaiser \\ Shahbaz ${ }^{3, *}$
}

* Corresponding Author: Muhammad Qaiser Shahbaz. Email: mkmohamad@kau.edu.sa

1. Department of Statistics, Faculty of Science, King Abdulaziz University, Jeddah, Kingdom of Saudi Arabia; jdarwish@kau.edu.sa 2. Department of Statistics, Faculty of Science, King Abdulaziz University, Jeddah, Kingdom of Saudi Arabia; lturk@kau.edu.sa

3. Department of Statistics, Faculty of Science, King Abdulaziz University, Jeddah, Kingdom of Saudi Arabia; mkmohamad@kau.edu.sa

\begin{abstract}
The bivariate distributions are useful in simultaneous modeling of two random variables. These distributions provide a way of modeling complex joint phenomenon. In this article, a new bivariate distribution is proposed which is known as the bivariate transmuted $\operatorname{Burr}(B T B)$ distribution. This new bivariate distribution is extension of the univariate transmuted Burr (TB) distribution to two variables. The proposed BTB distribution is explored in detail and the marginal and conditional distributions for the distribution are obtained. Joint and conditional moments alongside hazard rate functions are obtained. The maximum likelihood estimation (MLE) for the parameters of the $B T B$ distribution is also done. Finally, real data application of the $B T B$ distribution is given. It is observed that the proposed $B T B$ distribution is a suitable fit for the data used.
\end{abstract}

Key Words: Transmuted Distributions; Bivariate Distributions; Burr Distribution; Dependence Function; Maximum Likelihood Estimation.

Mathematical Subject Classification: 62E15, 62F10, 62H10.

\section{Introduction}

The extension and modification of probability distributions has been an interesting area within the domain of statistics. The probability distributions are extended and/or modified to have much wider applicability. The transmuted distributions, proposed by Shaw (2007), extend any baseline distribution by adding a new parameter to any baseline distribution with cumulative distribution function $(c d f) G(x)$. The $c d f$ of transmuted family of distributions is given as

$$
F(x)=G(x)+\lambda G(x)[1-G(x)] ; x \in \square,
$$

where $\lambda$ is the transmutation parameter, such that $|\lambda| \leq 1$. Alzaatreh et al. (2013) have proposed a general method of extending any baseline distribution $G(x)$ by using some other probability distribution $r(t)$ and the resulting family is referred to as the $T-X$ family of distributions. The $c d f$ of $T-X$ family of distributions is given as

$$
F_{T-X}(x)=\int_{a}^{W[G(x)]} r(t) d t ; a \leq t \leq b, x \in \square,
$$


where $W[G(x)]$ is some absolutely continuous function of $G(x)$ such that $W[0] \rightarrow a$ and $W[1] \rightarrow b$. Alizadeh et al. (2017) have shown that the transmuted family of distributions can be obtained as a member of $T-X$ family of distributions by using $r(t)=1+\lambda-2 \lambda t ; 0 \leq t \leq 1$ and $W[G(x)]=G(x)$ in (1). The $T-X$ and transmuted families of distributions have been used by several authors to propose the new univariate distributions, see for example Tahir and Nadarajah (2015) and Rehman et al. (2020).

Several situations arise where joint modeling of two random variables is required and in such cases a bivariate distribution is needed. The development in the bivariate families of distributions has been slightly new. Sarabia et al. (2014) have used the bivariate beta distribution as a baseline distribution to propose the bivariate beta generated family of distributions. Recently, Ganji et al. (2018) and Darwish et al. (2021) have proposed a bivariate extension of the $T$ $X$ family of distributions. The joint $c d f$ of the proposed bivariate family of distributions is

$$
F_{X, Y}(x, y)=\int_{a_{2}}^{W_{2}\left[G_{2}(y)\right]} \int_{a_{1}}^{W_{1}\left[G_{1}(x)\right]} r\left(u_{1}, u_{2}\right) d u_{1} d u_{2} ; a_{1} \leq u_{1} \leq b_{1}, a_{2} \leq u_{2} \leq b_{2},
$$

where $W_{1}\left[G_{1}(x)\right]$ and $W_{2}\left[G_{2}(y)\right]$ have usual properties. Derwish et al. (2021) have further argued that a simpler version of the bivariate $T-X$ family of distributions, when $u_{1}$ and $u_{2}$ have support on [0,1], is given as

$$
F_{X, Y}(x, y)=\int_{0}^{G_{2}(y)} \int_{0}^{G_{1}(x)} r\left(u_{1}, u_{2}\right) d u_{1} d u_{2} .
$$

Darwish et al. (2021) have used

$$
r\left(u_{1}, u_{2}\right)=1+\lambda_{1}\left(1-2 u_{1}\right)+\lambda_{2}\left(1-2 u_{2}\right)+2 \lambda_{3}\left(1-u_{1}-u_{2}\right)
$$

in (2) to propose the bivariate transmuted family of distributions. The $c d f$ of the proposed family of distributions is

$$
F_{X, Y}(x, y)=G_{1}(x) G_{2}(y)\left[1+\left(\lambda_{1}+\lambda_{3}\right)\left\{1-G_{1}(x)\right\}+\left(\lambda_{2}+\lambda_{3}\right)\left\{1-G_{2}(y)\right\}\right],(x, y) \in \square^{2}
$$

where $G_{1}(x)$ and $G_{2}(y)$ are $c d f$ 's of any baseline distributions and $\left(\lambda_{1}, \lambda_{2}, \lambda_{3}\right)$ are the transmutation parameters such that $\left(\lambda_{1}, \lambda_{2}, \lambda_{3}\right) \in[-1,1]$ and $-1 \leq \lambda_{1}+\lambda_{3} \leq 1$ and $-1 \leq \lambda_{2}+\lambda_{3} \leq 1$.

The probability density function ( $p d f)$ corresponding to (3) is

$$
f_{X, Y}(x, y)=g_{1}(x) g_{2}(y)\left[1+\left(\lambda_{1}+\lambda_{3}\right)\left\{1-2 G_{1}(x)\right\}+\left(\lambda_{2}+\lambda_{3}\right)\left\{1-2 G_{2}(y)\right\}\right],(x, y) \in \square^{2},
$$

where $g_{1}(x)$ and $g_{2}(y)$ are density functions corresponding to $G_{1}(x)$ and $G_{2}(y)$.

The transmuted family of distribution has been used by various authors to propose various transmuted distributions. One of these transmuted distributions is the transmuted Burr distribution which has been proposed by using the Burr distribution; Burr (1942); as a baseline distribution in the transmuted family of distributions. For more details on Burr Burr distribution one can refer to Tadikamalla (1980) and Kumar (2017). The details on transmuted Burr distribution can be found in Al-Khazaleh (2016); Maurya et al. (2017) and Afify et al. (2018).

In this article we have extended the transmuted Burr distribution to the bivariate case by using the bivariate transmuted family of distributions given in (3). The proposed distribution is named as the bivariate transmuted Burr (BTB) distribution.

The paper is organized as follows. The $B T B$ is proposed in next section. In Section 3, we have studied some important statistical properties of the proposed bivariate distribution. The estimation of the model parameters is discussed in Section 4. In Section 5, we have applied the proposed BTB distribution on real data set. Finally, the conclusions are included in Section 6.

\section{Bivariate Transmuted Burr Distribution}

In this section, we have proposes the $B T B$ distribution by using the following $c d f$ 's of Burr distribution

$$
G_{1}(x)=1-\left(1+x^{c}\right)^{-k} \text { and } G_{2}(y)=1-\left(1+y^{c}\right)^{-k} ; x, y, c, k>0
$$

in (3). The joint $c d f$ of proposed $B T B$ distribution is

$$
F_{X, Y}(x, y)=\left[1-\left(1+x^{c}\right)^{-k}\right]\left[1-\left(1+y^{c}\right)^{-k}\right]\left[1+\left(\lambda_{1}+\lambda_{3}\right)\left(1+x^{c}\right)^{-k}+\left(\lambda_{2}+\lambda_{3}\right)\left(1+y^{c}\right)^{-k}\right],
$$

for $(x, y)>0,\left(\lambda_{1}, \lambda_{2}, \lambda_{3}\right) \in[-1,1], k>0$ and $c>0$.

The density function of $B T B$ distribution corresponding to (6) is 


$$
f_{X, Y}(x, y)=\frac{c^{2} k^{2}(x y)^{c-1}}{\left[\left(1+x^{c}\right)\left(1+y^{c}\right)\right]^{k+1}}\left[1-\left(\lambda_{1}+\lambda_{2}+2 \lambda_{3}\right)+\frac{2\left(\lambda_{1}+\lambda_{3}\right)}{\left(1+x^{c}\right)^{k}}+\frac{2\left(\lambda_{2}+\lambda_{3}\right)}{\left(1+y^{c}\right)^{k}}\right] .
$$

Some properties of the proposed BTB distribution will be studied in the following section.

\section{Mathematical Properties}

In this section some properties of the proposed $B T B$ are studied. These include marginal and conditional distributions, conditional moments, product and ratio moments, bivariate reliability and bivariate hazard rate functions.

\subsection{The Marginal and Conditional Distributions}

The marginal $c d f$ of $X$ and $Y$ are immediately written from (4) as

$$
F_{T B}(x)=\left[1-\left(1+x^{c}\right)^{-k}\right]\left[1+\left(\lambda_{1}+\lambda_{3}\right)\left(1+x^{c}\right)^{-k}\right]
$$

and

$$
F_{T B}(y)=\left[1-\left(1+y^{c}\right)^{-k}\right]\left[1+\left(\lambda_{2}+\lambda_{3}\right)\left(1+y^{c}\right)^{-k}\right] .
$$

The marginal density functions of $X$ and $Y$ are given as

$$
f_{T B}(x)=\frac{c k x^{c-1}}{\left(1+x^{c}\right)^{k+1}}\left[1-\left(\lambda_{1}+\lambda_{3}\right)+2\left(\lambda_{1}+\lambda_{3}\right)\left(1+x^{c}\right)^{-k}\right]
$$

and

$$
f_{T B}(y)=\frac{c k y^{c-1}}{\left(1+y^{c}\right)^{k+1}}\left[1-\left(\lambda_{2}+\lambda_{3}\right)+2\left(\lambda_{2}+\lambda_{3}\right)\left(1+y^{c}\right)^{-k}\right] .
$$

We can see that the marginal distributions are transmuted Burr with specific transmutation parameters as given by Maurya et al. (2017).

The conditional pdf of $X$ given $Y=y$ for the $B T B$ distribution is obtained as

$$
f_{X \mid Y}(x \mid y)=\frac{c k x^{c-1}}{\Delta_{1}(y)\left(1+x^{c}\right)^{k+1}}\left[1-\left(\lambda_{1}+\lambda_{2}+2 \lambda_{3}\right)+\frac{2\left(\lambda_{1}+\lambda_{3}\right)}{\left(1+x^{c}\right)^{k}}+\frac{2\left(\lambda_{2}+\lambda_{3}\right)}{\left(1+y^{c}\right)^{k}}\right],
$$

where $\Delta_{1}(y)=1-\left(\lambda_{2}+\lambda_{3}\right)+2\left(\lambda_{2}+\lambda_{3}\right)\left(1+y^{c}\right)^{-k}$. Similarly, the conditional distribution of $Y$ given $X=x$ is written as

$$
f_{Y \mid X}(y \mid x)=\frac{c k y^{c-1}}{\Delta_{2}(x)\left(1+y^{c}\right)^{k+1}}\left[1-\left(\lambda_{1}+\lambda_{2}+2 \lambda_{3}\right)+\frac{2\left(\lambda_{1}+\lambda_{3}\right)}{\left(1+x^{c}\right)^{k}}+\frac{2\left(\lambda_{2}+\lambda_{3}\right)}{\left(1+y^{c}\right)^{k}}\right],
$$

where $\Delta_{2}(x)=1-\left(\lambda_{1}+\lambda_{3}\right)+2\left(\lambda_{1}+\lambda_{3}\right)\left(1+x^{c}\right)^{-k}$.

The conditional distributions are useful in obtaining the conditional moments of the distribution which will be obtained in the following subsection.

\subsection{The Conditional Moments}

In the following we will obtain the conditional moments for the conditional distributions given in (8) and (9). The $r$ th conditional moment of $X$ given $Y=y$ is defined as

$$
\mu_{X \mid y}^{r}=E\left(X^{r} \mid y\right)=\int_{-\infty}^{\infty} x^{r} f_{X \mid Y}(x \mid y) d x .
$$

Using (8) in above equation we have

$$
\mu_{X \mid y}^{r}=E\left(X^{r} \mid y\right)=\frac{1}{\Delta_{1}(y)} \int_{0}^{\infty} x^{r} \frac{c k x^{c-1}}{\left(1+x^{c}\right)^{k+1}}\left[1-\left(\lambda_{1}+\lambda_{2}+2 \lambda_{3}\right)+\frac{2\left(\lambda_{1}+\lambda_{3}\right)}{\left(1+x^{c}\right)^{k}}+\frac{2\left(\lambda_{2}+\lambda_{3}\right)}{\left(1+y^{c}\right)^{k}}\right] d x
$$

Simplifying, the $r$ th conditional moment of $X$ given $Y=y$ is

$$
\mu_{X \mid y}^{r}=\frac{1}{\Delta_{1}(y)} k B\left(\frac{r}{c}+1, k-\frac{r}{c}\right)\left[\left\{1-(b+d)+2 d\left(1+y^{c}\right)^{-k}\right\}+2 b \frac{B(r / c+1,2 k-r / c)}{B(r / c+1, k-r / c)}\right],
$$


where $B(a, b)$ is the complete Beta function, $b=\lambda_{1}+\lambda_{3}$ and $d=\lambda_{2}+\lambda_{3}$. Again the sth conditional moment of $Y$ given $X=x$ is obtained by using

$$
\mu_{Y \mid x}^{s}=E\left(Y^{s} \mid x\right)=\int_{-\infty}^{\infty} y^{s} f_{Y \mid X}(y \mid x) d y .
$$

Using (9) in above equation we have

$$
\mu_{Y \mid x}^{s}=\int_{0}^{\infty} y^{s} \frac{c k y^{c-1}}{\Delta_{2}(x)\left(1+y^{c}\right)^{k+1}}\left[1-\left(\lambda_{1}+\lambda_{2}+2 \lambda_{3}\right)+\frac{2\left(\lambda_{1}+\lambda_{3}\right)}{\left(1+x^{c}\right)^{k}}+\frac{2\left(\lambda_{2}+\lambda_{3}\right)}{\left(1+y^{c}\right)^{k}}\right] d y .
$$

Solving above integral, the sth conditional moment of $Y$ given $X=x$ is

$$
\mu_{Y \mid x}^{s}=\frac{1}{\Delta_{2}(x)} k B\left(\frac{s}{c}+1, k-\frac{s}{c}\right)\left[\left\{1-(b+d)+2 b\left(1+x^{c}\right)^{-k}\right\}+2 d \frac{B(s / c+1,2 k-s / c)}{B(s / c+1, k-s / c)}\right],
$$

where $b$ and $d$ are defined earlier.

The conditional moments are useful to obtain the conditional mean and conditional variance of the distribution. The product moments are useful to obtain the measure of dependence between two random variables. In the following subsection we will obtain the product and ratio moments for the $B T B$.

\subsection{The Product and Ratio Moments}

The $(r, s)$ th product moment for a joint density function is defined as

$$
\mu_{r, s}^{\prime}=E\left(X^{r} Y^{s}\right)=\int_{-\infty}^{\infty} \int_{-\infty}^{\infty} x^{r} y^{s} f_{X, Y}(x, y) d x d y .
$$

Using the joint density function of $B T B$ distribution, the expression for $(r, s)$ th product moment is

$$
\mu_{r, s}^{\prime}=E\left(X^{r} Y^{s}\right)=\int_{0}^{\infty} \int_{0}^{\infty} x^{r} y^{s} \frac{c^{2} k^{2}(x y)^{c-1}}{\left[\left(1+x^{c}\right)\left(1+y^{c}\right)\right]^{k+1}}\left[1-\left(\lambda_{1}+\lambda_{2}+2 \lambda_{3}\right)+\frac{2\left(\lambda_{1}+\lambda_{3}\right)}{\left(1+x^{c}\right)^{k}}+\frac{2\left(\lambda_{2}+\lambda_{3}\right)}{\left(1+y^{c}\right)^{k}}\right] d x d y .
$$

Simplifying above, the expression of product moments for the $B T B$ distribution is

$$
\begin{aligned}
\mu_{r, s}^{\prime}=k^{2} B\left(\frac{r}{c}+1, k\right. & \left.-\frac{r}{c}\right) B\left(\frac{s}{c}+1, k-\frac{s}{c}\right)\left[\{1-(b+d)\}+\frac{2 b B(r / c+1,2 k-r / c)}{B(r / c+1, k-r / c)}\right. \\
& \left.+\frac{2 d B(s / c+1,2 k-s / c)}{B(s / c+1, k-s / c)}\right] .
\end{aligned}
$$

The product moments can be used to obtain the covariance and correlation coefficient between $X$ and $Y$ which are given in the Table 1 below. It can be seen that the correlation coefficient between $X$ and $Y$ for $B T B$ distribution is positive if $\left(\lambda_{1}+\lambda_{3}\right)$ and $\left(\lambda_{2}+\lambda_{3}\right)$ are of opposite signes and is negative otherwise. Also for fixed value of $k$, the correlation coefficient decreases with increase in $c$ and for fixed value of $c$ the correlation coefficient increases with increase in $k$.

The ratio moments for two variables can be defined in two ways. The first of these is computed as

$$
\mu_{r,-s}^{\prime}=E\left(X^{r} Y^{-s}\right)=\int_{-\infty}^{\infty} \int_{-\infty}^{\infty} x^{r} y^{-s} f_{X, Y}(x, y) d x d y
$$

which for the $B T B$ distribution is

$$
\mu_{r,-s}^{\prime}=E\left(X^{r} Y^{-s}\right)=\int_{0}^{\infty} \int_{0}^{\infty} x^{r} y^{-s} \frac{c^{2} k^{2}(x y)^{c-1}}{\left[\left(1+x^{c}\right)\left(1+y^{c}\right)\right]^{k+1}}\left[1-\left(\lambda_{1}+\lambda_{2}+2 \lambda_{3}\right)+\frac{2\left(\lambda_{1}+\lambda_{3}\right)}{\left(1+x^{c}\right)^{k}}+\frac{2\left(\lambda_{2}+\lambda_{3}\right)}{\left(1+y^{c}\right)^{k}}\right] d x d y .
$$

On simplification the expression for ratio moment $\mu_{r,-s}^{\prime}$ for $B T B$ distribution is 
Table 1: Correlation Coefficient for the Bivariate Transmuted Burr Distribution

\begin{tabular}{|c|c|c|c|c|c|c|c|c|c|}
\hline \multicolumn{10}{|c|}{$\lambda_{1}=-0.50, \lambda_{2}=-0.75, \lambda_{3}=-0.25$} \\
\hline \multirow[b]{2}{*}{$\boldsymbol{k}$} & \multicolumn{9}{|c|}{$c$} \\
\hline & 2.0 & 2.5 & 3.0 & 3.5 & 4.0 & 4.5 & 5.0 & 5.5 & 6.0 \\
\hline 2.0 & -0.1317 & -0.1691 & -0.1963 & -0.2169 & -0.2330 & -0.2460 & -0.2566 & -0.2654 & -0.2729 \\
\hline 2.5 & -0.1585 & -0.1936 & -0.2193 & -0.2387 & -0.2540 & -0.2662 & -0.2762 & -0.2846 & -0.2916 \\
\hline 3.0 & -0.1760 & -0.2098 & -0.2345 & -0.2533 & -0.2679 & -0.2797 & -0.2894 & -0.2974 & -0.3043 \\
\hline 3.5 & -0.1882 & -0.2212 & -0.2453 & -0.2636 & -0.2779 & -0.2894 & -0.2988 & -0.3066 & -0.3133 \\
\hline 4.0 & -0.1973 & -0.2297 & -0.2533 & -0.2713 & -0.2853 & -0.2966 & -0.3058 & -0.3135 & -0.3200 \\
\hline 4.5 & -0.2043 & -0.2362 & -0.2595 & -0.2772 & -0.2910 & -0.3021 & -0.3112 & -0.3188 & -0.3252 \\
\hline 5.0 & -0.2098 & -0.2414 & -0.2644 & -0.2819 & -0.2956 & -0.3066 & -0.3155 & -0.3230 & -0.3294 \\
\hline 5.5 & -0.2143 & -0.2456 & -0.2684 & -0.2857 & -0.2993 & -0.3102 & -0.3191 & -0.3265 & -0.3328 \\
\hline 6.0 & -0.2180 & -0.2491 & -0.2717 & -0.2889 & -0.3024 & -0.3131 & -0.3220 & -0.3293 & -0.3356 \\
\hline \multicolumn{10}{|c|}{$\lambda_{1}=0.75, \lambda_{2}=-0.50, \lambda_{3}=0.25$} \\
\hline & \multicolumn{9}{|c|}{$c$} \\
\hline $\boldsymbol{k}$ & 2.0 & 2.5 & 3.0 & 3.5 & 4.0 & 4.5 & 5.0 & 5.5 & 6.0 \\
\hline 2.0 & 0.1090 & 0.1093 & 0.1080 & 0.1064 & 0.1048 & 0.1033 & 0.1020 & 0.1008 & 0.0998 \\
\hline 2.5 & 0.1112 & 0.1098 & 0.1076 & 0.1056 & 0.1037 & 0.1021 & 0.1007 & 0.0994 & 0.0984 \\
\hline 3.0 & 0.1119 & 0.1096 & 0.1071 & 0.1048 & 0.1028 & 0.1011 & 0.0997 & 0.0984 & 0.0973 \\
\hline 3.5 & 0.1121 & 0.1093 & 0.1066 & 0.1042 & 0.1021 & 0.1004 & 0.0989 & 0.0976 & 0.0965 \\
\hline 4.0 & 0.1121 & 0.1090 & 0.1061 & 0.1036 & 0.1015 & 0.0998 & 0.0983 & 0.0970 & 0.0959 \\
\hline 4.5 & 0.1120 & 0.1087 & 0.1057 & 0.1032 & 0.1011 & 0.0993 & 0.0978 & 0.0965 & 0.0954 \\
\hline 5.0 & 0.1119 & 0.1084 & 0.1054 & 0.1028 & 0.1007 & 0.0989 & 0.0974 & 0.0961 & 0.0950 \\
\hline 5.5 & 0.1118 & 0.1082 & 0.1051 & 0.1025 & 0.1004 & 0.0986 & 0.0971 & 0.0958 & 0.0946 \\
\hline 6.0 & 0.1117 & 0.1080 & 0.1049 & 0.1023 & 0.1001 & 0.0983 & 0.0968 & 0.0955 & 0.0944 \\
\hline \multicolumn{10}{|c|}{$\lambda_{1}=-0.25, \lambda_{2}=0.75, \lambda_{3}=-0.50$} \\
\hline & \multicolumn{9}{|c|}{$c$} \\
\hline $\boldsymbol{k}$ & 2.0 & 2.5 & 3.0 & 3.5 & 4.0 & 4.5 & 5.0 & 5.5 & 6.0 \\
\hline 2.0 & 0.0408 & 0.0483 & 0.0529 & 0.0559 & 0.0580 & 0.0595 & 0.0606 & 0.0615 & 0.0622 \\
\hline 2.5 & 0.0465 & 0.0527 & 0.0565 & 0.0589 & 0.0606 & 0.0618 & 0.0627 & 0.0634 & 0.0639 \\
\hline 3.0 & 0.0498 & 0.0553 & 0.0586 & 0.0607 & 0.0622 & 0.0632 & 0.0640 & 0.0646 & 0.0650 \\
\hline 3.5 & 0.0521 & 0.0570 & 0.0600 & 0.0619 & 0.0632 & 0.0642 & 0.0648 & 0.0653 & 0.0657 \\
\hline 4.0 & 0.0536 & 0.0582 & 0.0610 & 0.0628 & 0.0640 & 0.0648 & 0.0654 & 0.0659 & 0.0662 \\
\hline 4.5 & 0.0547 & 0.0591 & 0.0617 & 0.0634 & 0.0645 & 0.0653 & 0.0659 & 0.0663 & 0.0666 \\
\hline 5.0 & 0.0556 & 0.0598 & 0.0623 & 0.0639 & 0.0649 & 0.0657 & 0.0662 & 0.0666 & 0.0669 \\
\hline 5.5 & 0.0563 & 0.0603 & 0.0627 & 0.0642 & 0.0653 & 0.0660 & 0.0665 & 0.0668 & 0.0671 \\
\hline 6.0 & 0.0569 & 0.0608 & 0.0631 & 0.0645 & 0.0655 & 0.0662 & 0.0667 & 0.0670 & 0.0673 \\
\hline \multicolumn{10}{|c|}{$\lambda_{1}=0.25, \lambda_{2}=0.50, \lambda_{3}=0.25$} \\
\hline & \multicolumn{9}{|c|}{$c$} \\
\hline$k$ & 2.0 & 2.5 & 3.0 & 3.5 & 4.0 & 4.5 & 5.0 & 5.5 & 6.0 \\
\hline 2.0 & -0.1500 & -0.1553 & -0.1549 & -0.1530 & -0.1506 & -0.1484 & -0.1462 & -0.1443 & -0.1426 \\
\hline 2.5 & -0.1563 & -0.1569 & -0.1545 & -0.1516 & -0.1487 & -0.1460 & -0.1437 & -0.1416 & -0.1398 \\
\hline 3.0 & -0.1586 & -0.1570 & -0.1536 & -0.1502 & -0.1470 & -0.1442 & -0.1418 & -0.1397 & -0.1378 \\
\hline 3.5 & -0.1595 & -0.1566 & -0.1527 & -0.1490 & -0.1457 & -0.1428 & -0.1403 & -0.1382 & -0.1363 \\
\hline 4.0 & -0.1598 & -0.1562 & -0.1519 & -0.1480 & -0.1446 & -0.1417 & -0.1392 & -0.1370 & -0.1351 \\
\hline 4.5 & -0.1599 & -0.1557 & -0.1512 & -0.1472 & -0.1437 & -0.1408 & -0.1383 & -0.1361 & -0.1342 \\
\hline 5.0 & -0.1598 & -0.1553 & -0.1507 & -0.1465 & -0.1430 & -0.1401 & -0.1375 & -0.1354 & -0.1335 \\
\hline 5.5 & -0.1597 & -0.1549 & -0.1501 & -0.1460 & -0.1424 & -0.1395 & -0.1369 & -0.1347 & -0.1329 \\
\hline 6.0 & -0.1596 & -0.1546 & -0.1497 & -0.1455 & -0.1419 & -0.1389 & -0.1364 & -0.1342 & -0.1323 \\
\hline
\end{tabular}




$$
\begin{gathered}
\mu_{r,-s}^{\prime}=k^{2} B\left(\frac{r}{c}+1, k-\frac{r}{c}\right) B\left(1-\frac{s}{c}, k+\frac{s}{c}\right)\left[1-(b+d)+\frac{2 b B(r / c+1,2 k-r / c)}{B(r / c+1, k-r / c)}\right. \\
\left.+\frac{2 d B(1-s / c, 2 k+s / c)}{B(1-s / c, k+s / c)}\right] ; s<c .
\end{gathered}
$$

Similarly, the expression for the ratio moments $\mu_{-r, s}^{\prime}$ for the $B T B$ distribution is

$$
\begin{gathered}
\mu_{-r, s}^{\prime}=k^{2} B\left(1-\frac{r}{c}, k+\frac{r}{c}\right) B\left(\frac{s}{c}+1, k-\frac{s}{c}\right)\left[1-(b+d)+\frac{2 b B(1-r / c, 2 k+r / c)}{B(1-r / c, k+r / c)}\right. \\
\left.+\frac{2 d B(s / c+1,2 k-s / c)}{B(s / c+1, k-s / c)}\right] .
\end{gathered}
$$

\subsection{The Bivariate Dependence Function}

The bivariate dependence function for a continuous bivariate distribution is defined by Holland and Wang (1987) as

$$
\gamma(x, y)=\frac{\partial^{2}}{\partial x \partial y} \ln f(x, y) .
$$

It can be easily seen that the dependence function for the bivariate transmuted family of distributions is

$$
\gamma(x, y)=-\frac{4 b d g_{1}(x) g_{2}(y)}{\left[1+b\left\{1-2 G_{1}(x)\right\}+d\left\{1-2 G_{2}(y)\right\}\right]^{2}} .
$$

Using the marginal density and distribution functions of the Burr distribution in above equation, the dependence function for the $B T B$ distribution is

$$
\gamma(x, y)=-\frac{4 b d c^{2} k^{2}(x y)^{c-1}\left[\left(1+x^{c}\right)\left(1+y^{c}\right)\right]^{-(k+1)}}{\left[1-(b+d)+2 b\left(1+x^{c}\right)^{-k}+2 d\left(1+y^{c}\right)^{-k}\right]^{2}},
$$

where $b$ and $d$ are defined earlier. It is to be noted that $\gamma(x, y)>0$ if $b$ and $d$ are of opposite sign and $\gamma(x, y)<0$ otherwise. This coincides with the values of correlation coefficients given in Table 1 above. The dependence function can be computed for given values of the parameters.

\subsection{The Bivariate Reliability and Hazard Rate Functions}

The bivariate reliability function is defined as

$$
R(x, y)=1-\left[F_{X}(x)+F_{Y}(y)-F_{X, Y}(x, y)\right] .
$$

Using joint and marginal $c d f$ 's, the bivariate reliability function for the $B T B$ distribution is

$$
R(x, y)=\left(1+x^{c}\right)^{-k}\left(1+y^{c}\right)^{-k}\left[1-(b+d)+b\left(1+x^{c}\right)^{-k}+d\left(1+y^{c}\right)^{-k}\right]
$$

The bivariate hazard rate function is defined as

$$
h(x, y)=\frac{f_{X, Y}(x, y)}{R(x, y)} .
$$

Now, using the joint density function and joint reliability function of the BTB distribution, from (7) and (16), the bivariate hazard rate function for the $B T B$ distribution is

$$
h(x, y)=\frac{c^{2} k^{2}(x y)^{c-1}\left[1-(b+d)+2 b\left(1+x^{c}\right)^{-k}+2 d\left(1+y^{c}\right)^{-k}\right]}{\left(1+x^{c}\right)\left(1+y^{c}\right)\left[1-(b+d)+b\left(1+x^{c}\right)^{-k}+d\left(1+y^{c}\right)^{-k}\right]} .
$$

The plot of hazard rate function for different choices of the parameters is shown in Figure- 1 below. The graph shows that the hazard rate function is decreasing for $c<1$. For $(k, c)>1$, the hazard rate first increases and then decreases for all the combinations of $\left(\lambda_{1}, \lambda_{2}, \lambda_{3}\right)$. The decrease in the hazard rate function is fast when $\left(\lambda_{1}, \lambda_{2}\right)>0$ and is slow for $\left(\lambda_{1}, \lambda_{2}\right)<0$. 
Figure 1: The hazard rate function of the bivariate transmuted Burr distribution

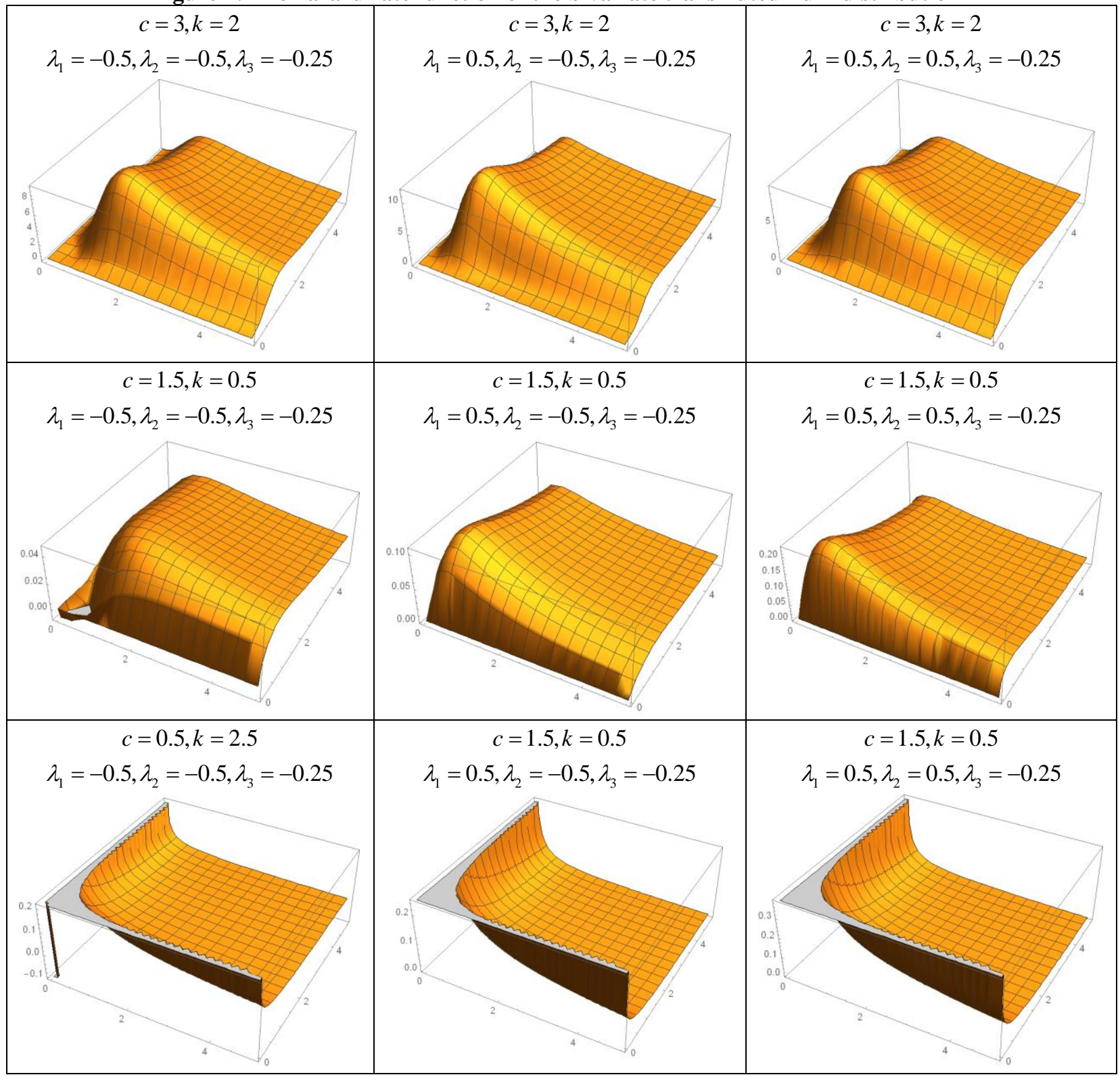

\section{Parameter Estimation}

The parameters of $B T B$ distribution are estimated by using the maximum likelihood method under the assumption that all the parameters are unknown. For this, let $X_{1}, X_{2}, \ldots, X_{n}$ be a random sample of size $n$ from the $B T B$ distribution. The likelihood function is

$$
\begin{aligned}
L=c^{2 n} k^{2 n} & \prod_{i=1}^{n}\left(x_{i} y_{i}\right)^{c-1} \prod_{i=1}^{n}\left\{\left(1+x_{i}^{c}\right)\left(1+y_{i}^{c}\right)\right\}^{-(k+1)} \\
& \times \prod_{i=1}^{k}\left[1-\left(\lambda_{1}+\lambda_{2}+2 \lambda_{3}\right)+2\left(\lambda_{1}+\lambda_{3}\right)\left(1+x_{i}^{c}\right)^{-k}+2\left(\lambda_{2}+\lambda_{3}\right)\left(1+y_{i}^{c}\right)^{-k}\right] .
\end{aligned}
$$

The log-likelihood function is

$$
\begin{aligned}
\ell=2 n \ln c & +2 n \ln k+(c-1) \sum_{i=1}^{n} \ln \left(x_{i} y_{i}\right)-(k+1) \sum_{i=1}^{n} \ln \left\{\left(1+x_{i}^{c}\right)\left(1+y_{i}^{c}\right)\right\} \\
+ & \sum_{i=1}^{n} \ln \left[1-\left(\lambda_{1}+\lambda_{2}+2 \lambda_{3}\right)+2\left(\lambda_{1}+\lambda_{3}\right)\left(1+x_{i}^{c}\right)^{-k}+2\left(\lambda_{2}+\lambda_{3}\right)\left(1+y_{i}^{c}\right)^{-k}\right] .
\end{aligned}
$$


The maximum likelihood estimators of $k, c, \lambda_{1}, \lambda_{2}$ and $\lambda_{3}$ are obtained by differentitaing $\log$-likelihood function with respect to unknown parameters, equating the derivaties to zero and solving the resulting equations. The derivaties of log-likelihood function with respect to the unknown parameters are

$$
\begin{aligned}
& \frac{\partial \ell}{\partial k}=\frac{2 n}{k}-\sum_{i=1}^{n} \ln \left\{\left(1+x_{i}^{c}\right)\left(1+y_{i}^{c}\right)\right\} \\
& -2 \sum_{i=1}^{n} \frac{\left(\lambda_{1}+\lambda_{3}\right)\left(1+x_{i}^{c}\right)^{-k} \ln \left(1+x_{i}^{c}\right)+\left(\lambda_{2}+\lambda_{3}\right)\left(1+y_{i}^{c}\right)^{-k} \ln \left(1+y_{i}^{c}\right)}{\left[1-\left(\lambda_{1}+\lambda_{2}+2 \lambda_{3}\right)+2\left(\lambda_{1}+\lambda_{3}\right)\left(1+x_{i}^{c}\right)^{-k}+2\left(\lambda_{2}+\lambda_{3}\right)\left(1+y_{i}^{c}\right)^{-k}\right]} \\
& \frac{\partial \ell}{\partial c}=\frac{2 n}{c}+\sum_{i=1}^{n} \ln \left(x_{i} y_{i}\right)-(k+1) \sum_{i=1}^{n} \frac{x_{i}^{c} \ln x_{i}\left(1+y_{i}^{c}\right)+y_{i}^{c} \ln y_{i}\left(1+x_{i}^{c}\right)}{\left(1+x_{i}^{c}\right)\left(1+y_{i}^{c}\right)} \\
& -2 k \sum_{i=1}^{n} \frac{\left(\lambda_{1}+\lambda_{3}\right)\left(1+x_{i}^{c}\right)^{-(k+1)} x_{i}^{c} \ln x_{i}+\left(\lambda_{2}+\lambda_{3}\right)\left(1+y_{i}^{c}\right)^{-(k+1)} y_{i}^{c} \ln y_{i}}{\left[1-\left(\lambda_{1}+\lambda_{2}+2 \lambda_{3}\right)+2\left(\lambda_{1}+\lambda_{3}\right)\left(1+x_{i}^{c}\right)^{-k}+2\left(\lambda_{2}+\lambda_{3}\right)\left(1+y_{i}^{c}\right)^{-k}\right]} \\
& \frac{\partial \ell}{\partial \lambda_{1}}=\sum_{i=1}^{n} \frac{2\left(1+x_{i}^{c}\right)^{-k}-1}{\left[1-\left(\lambda_{1}+\lambda_{2}+2 \lambda_{3}\right)+2\left(\lambda_{1}+\lambda_{3}\right)\left(1+x_{i}^{c}\right)^{-k}+2\left(\lambda_{2}+\lambda_{3}\right)\left(1+y_{i}^{c}\right)^{-k}\right]} \\
& \frac{\partial \ell}{\partial \lambda_{2}}=\sum_{i=1}^{n} \frac{2\left(1+y_{i}^{c}\right)^{-k}-1}{\left[1-\left(\lambda_{1}+\lambda_{2}+2 \lambda_{3}\right)+2\left(\lambda_{1}+\lambda_{3}\right)\left(1+x_{i}^{c}\right)^{-k}+2\left(\lambda_{2}+\lambda_{3}\right)\left(1+y_{i}^{c}\right)^{-k}\right]}
\end{aligned}
$$

and

$$
\frac{\partial \ell}{\partial \lambda_{3}}=\sum_{i=1}^{n} \frac{2\left[\left(1+x_{i}^{c}\right)^{-k}+\left(1+y_{i}^{c}\right)^{-k}-1\right]}{\left[1-\left(\lambda_{1}+\lambda_{2}+2 \lambda_{3}\right)+2\left(\lambda_{1}+\lambda_{3}\right)\left(1+x_{i}^{c}\right)^{-k}+2\left(\lambda_{2}+\lambda_{3}\right)\left(1+y_{i}^{c}\right)^{-k}\right]}
$$

The maximum likelihood estimators of parameter vector $\Theta=\left(k, c, \lambda_{1}, \lambda_{2}, \lambda_{3}\right)$ will be obtained by equating above derivatives to zero and numerically solving the resulting equations.

\section{Real Data Application}

In this section, we have applied the $B T B$ distribution for modeling of a real data set. The data has been modeled by using the proposed $B T B$ distribution alongside three more distributions. The other distributions that we have used in the study are bivariate Burr (BB) by Durling (1975), Gumbel bivariate Burr (GBB) and Gumbel bivariate Lomax (GBL). The distributions have fitted by obtaining the $M L E$ 's of the parameters. The MLE's have been obtained by using the R-package "maxLik" by Henningsen and Toomet (2011). In order to assess the performance of the proposed $B T B$ distribution with the competing distributions we have obtained Akaike's information criterion $(A I C)$ and Bayesian information criterion $(B I C)$ for each fitted model.

In the data set $X$ represents the Force and $Y$ represents the Breaking Strength of fluid. Table 2 gives some descriptive statistics of the data.

Table 2: Summary Statistics for Dataset

\begin{tabular}{ccccccc}
\hline \multicolumn{8}{c}{ Min. } & $Q_{1}$ & Median & Mean & $Q_{3}$ & Max. \\
\hline $\mathrm{X}$ & 0.6655 & 11.8092 & 22.3475 & 24.8074 & 34.3035 & 60.9240 \\
$\mathrm{Y}$ & 1.4331 & 8.5957 & 12.4628 & 15.3732 & 18.9041 & 41.4689 \\
\hline
\end{tabular}

The results of MLEs are listed in Table 3 and computed values of AIC and BIC are given in Table 3. From Table 3, we can see that the proposed BTB distribution has smallest values of AIC and BIC and hence is considered as the best fit for the data. 
Table 3: MLEs and SEs for Selected Distributions

\begin{tabular}{cccc}
\hline Distribution & Parameter & Estimate & SE \\
\hline BTB & $k$ & 9.8129 & 8.3886 \\
& $c$ & 0.8827 & 8.3886 \\
& $\lambda_{1}$ & -0.1280 & 5.6453 \\
& $\lambda_{2}$ & -0.1280 & 14.5339 \\
& $\lambda_{3}$ & -0.8720 & 8.3886 \\
BB & & & \\
& $p$ & $4.161 \mathrm{e}-02$ & $1.231 \mathrm{e}-02$ \\
& $\alpha_{1}$ & $9.436 \mathrm{e}-03$ & $1.974 \mathrm{e}-02$ \\
& $\alpha_{2}$ & $2.489 \mathrm{e}-04$ & $5.095 \mathrm{e}-04$ \\
& $\beta_{1}$ & $9.860 \mathrm{e}+00$ & $2.029 \mathrm{e}+00$ \\
& $\beta_{2}$ & $1.288 \mathrm{e}+01$ & $2.649 \mathrm{e}+00$ \\
GBB & & & \\
& $k_{1}$ & $1.635 \mathrm{e}-07$ & $7.430 \mathrm{e}-04$ \\
& $k_{2}$ & $1.892 \mathrm{e}-07$ & $8.853 \mathrm{e}-04$ \\
& $c_{1}$ & $7.567 \mathrm{e}-02$ & $5.968 \mathrm{e}-02$ \\
& $c_{2}$ & $6.122 \mathrm{e}-02$ & $6.796 \mathrm{e}-02$ \\
& $\gamma$ & $1.000 \mathrm{e}+00$ & $2.391 \mathrm{e}-01$ \\
GBL & & & \\
& $k_{1}$ & 0.4836 & 0.0595 \\
& $k_{2}$ & 2.1915 & 1.2788 \\
& $\gamma$ & 1.0000 & 0.4741 \\
\hline
\end{tabular}

Table 4: Selection Criteria for Selected Distributions

\begin{tabular}{cccc}
\hline Distribution & LogLik & AIC & BIC \\
\hline BTB & 76.8980 & -143.7970 & -175.0395 \\
BB & -473.7103 & 957.4205 & 968.6631 \\
GBB & 48.5202 & -87.0404 & -118.2829 \\
GBL & -334.1630 & 674.3260 & 681.0715 \\
\hline
\end{tabular}

\section{Conclusions}

In this paper, we have introduced the $B T B$ distribution. Various properties of the proposed bivariate distribution have been studied. We have also applied the $B T B$ distribution on the real data set. We have seen that the proposed $B T B$ turned out to be the best fit for modeling of the data used.

\section{References}

1. Afify, A. Z., Cordeiro, G. M., Bourguignon, M. \& Ortega, E. (2018). Properties of the transmuted Burr XII distribution, regression and its applicarions. Journal of Data Science, 16(3), 485-510.

2. Al-Khazaleh, A. M. H. (2016). Transmuted Burr type XII distribution: A generalization of the Burr type XII distribution. International Mathematical Forum, 11, 547-556.

3. Alizadeh, M., Merovci, F., Hamedani, G.G., (2017). Generalized transmuted family of distributions: Properties and applications. Hacettepe J. Math. and Stat., 46, 645-667.

4. Alzaatreh, A., Lee, C. \& Famoye, F. (2013). A new method for generating families of continuous distributions. Metron, 71, 63-79.

5. Burr, I. W. (1942). Cumulative frequency functions. Annals of Mathematical Statistics, 13, 215-232.

6. Durling, F. C. (1975). The bivariate Burr distribution. In: Patil G.P., Kotz S., Ord J.K. (eds) A Modern Course on Statistical Distributions in Scientific Work. NATO Advanced Study Institutes Series (Series C-Mathematical and Physical Sciences), vol. 17. Springer, Dordrecht.

7. Darwish, J. A., Al turk, L. I. \& Shahbaz, M. Q. (2021). The bivariate transmuted family of distributions: Theory and applications. Computer Systems Science and Enginnering, In press.

8. Ganji, M., Bevrani, H. \& Golzar, N. H. (2018). A new method of generating continuous bivariate distribution families, Journal of Iranian Statistical Society, 17, 109-129.

9. Henningsen, A. \& Toomet, O. (2011). maxLik: A package for maximum likelihood estimation in R. Computational Statistics, 26, 443-458. 
10. Kumar, D. (2017). The Burr type XII distribution with some statistical properties. Journal of Data Science, 16, 509-534.

11. Maurya, R. K., Tripathi, Y. M. \& Rastogi, M. K. (2017). Transmuted Burr XII distribution. Journal of the Indian Society for Probability and Statistics, 18, 177-193.

12. Rahman, M.M., Al-Zahrani, B., Hanif Shahbaz, S., \& Shahbaz, M.Q., (2020). Transmuted distributions: a review. Pak. J. Stat. and Operation Research, 16, 83-94

13. Tadikamalla, P. R. (1980). A look at the Burr and related distributions. International Statistical Review, 48, 337344.

14. Tahir, M. H. \& Nadarajah, S. (2015). Parameter induction in continuous univariate distributions: Well-established $\mathrm{G}$ families, Ann. Braz. Acad. Sci., 87, 539-568 